\title{
Genome-wide identification of hypoxia-induced enhancer regions
}

Nick Kamps-Hughes, Jessica L Preston, Melissa A Randel, Eric A Johnson

Here we present a genome-wide method for de novo identification of enhancer regions. This approach enables massively parallel empirical investigation of DNA sequences that mediate transcriptional activation and provides a platform for discovery of regulatory modules capable of driving context-specific gene expression. The method links fragmented genomic DNA to the transcription of randomer molecule identifiers and measures the functional enhancer activity of the library by massively parallel sequencing. We transfected a Drosophila melanogaster library into S2 cells in normoxia and hypoxia, and assayed 4,599,881 genomic DNA fragments in parallel. The locations of the enhancer regions strongly correlate with genes up-regulated after hypoxia and previously described enhancers. Novel enhancer regions were identified and integrated with RNAseq data and transcription factor motifs to describe the hypoxic response on a genome-wide basis as a complex regulatory network involving multiple stress-response pathways. This work provides a novel method for high-throughput assay of enhancer activity and the genomescale identification of 31 hypoxia-activated enhancers in Drosophila. 


\section{Genome-wide identification of hypoxia-induced enhancer regions}

2

3 Nick Kamps-Hughes ${ }^{1}$, Jessica L Preston ${ }^{1}$, Melissa A Randel ${ }^{1}$ and Eric A Johnson ${ }^{1}$

$4 \quad{ }^{1}$ Institute of Molecular Biology, University of Oregon, Eugene, Oregon, 97403, USA

5

6 Corresponding author:

7 Nick Kamps-Hughes

8590 Merritt Ave \#32, Oakland, CA, 94610, USA

9 Email address: nkampshughes@gmail.com 
11

12

\section{Genome-wide identification of hypoxia-induced enhancer regions}

Nick Kamps-Hughes ${ }^{1}$, Jessica L Preston ${ }^{1}$, Melissa A Randel ${ }^{1}$ and Eric A Johnson ${ }^{1}$

${ }^{1}$ Institute of Molecular Biology, University of Oregon, Eugene, Oregon, 97403, USA

\section{ABSTRACT}

Here we present a genome-wide method for de novo identification of enhancer regions. This approach enables massively parallel empirical investigation of DNA sequences that mediate transcriptional activation and provides a platform for discovery of regulatory modules capable of driving context-specific gene expression. The method links fragmented genomic DNA to the transcription of randomer molecule identifiers and measures the functional enhancer activity of the library by massively parallel sequencing. We transfected a Drosophila melanogaster library into S2 cells in normoxia and hypoxia, and assayed 4,599,881 genomic DNA fragments in parallel. The locations of the enhancer regions strongly correlate with genes up-regulated after hypoxia and previously described enhancers. Novel enhancer regions were identified and integrated with RNAseq data and transcription factor motifs to describe the hypoxic response on a genome-wide basis as a complex regulatory network involving multiple stress-response pathways. This work provides a novel method for high-throughput assay of enhancer activity and the genome-scale identification of 31 hypoxia-activated enhancers in Drosophila.

\section{INTRODUCTION}

Gene expression is differently regulated in different cell types and in response to changes to environmental conditions. This regulation is achieved in part by the activity of transcriptional enhancers $^{1-5}$, specific DNA sequences that bind transcription factors to control the rate of transcription initiated at nearby promoters. Even for relatively simple processes, such as the acute response to changes in oxygen availability, the identification and characterization of the enhancers used to shift the network of gene expression to a new mode remains limited.

The transcription factor hypoxia-inducible factor-1 (HIF-1) is directly inhibited by the presence of cellular oxygen via protein degradation of the HIF-1 $\alpha$ subunit $^{6}$. Once stabilized, HIF-1 $\alpha$ moves to the nucleus and up-regulates the transcription of target genes. Although HIF-1 remains 
43 a central regulator in models of how cells respond after experiencing low oxygen ${ }^{7,8}$, more

44 recently other transcription factors have been implicated in the hypoxic response in a complex network of regulatory events. For example, the immunity response transcription factor NF-KB is also activated by hypoxia and regulates the transcription of HIF-1 ${ }^{9,10}$, while HIF-1 appears to play a reciprocal role in the regulation of NF-kB targets ${ }^{11}$. Likewise, HIF-1 sensitizes the heat shock response by directly regulating heat shock factor (HSF) transcription during hypoxia. Thus, the broader picture that has emerged is that the stress response transcription factor pathways are not isolated regulatory units but rather cooperate and co-opt each other to modify the cell's functions in a complex manner.

High-throughput sequencing tools have become widespread in gene expression studies ${ }^{12-14}$. For example, RNAseq has become a powerful tool for analyzing differential gene expression by quantifying the RNA abundance of the transcriptome. However, RNAseq does not provide empirical information about the regulatory events leading to a change in transcript abundance. ChIPseq provides information about where transcription factors bind to the genome, but binding events do not always result in an active enhancer or change in the rate of transcription. Another sequencing strategy assays open chromatin conformations ${ }^{15}$ as a reliable proxy for enhancers. However, until recently the typical functional assay for enhancers was to clone the putative regulator upstream of a reporter gene driven by a minimal promoter.

Several next-generation sequencing-based methods have been used to dissect the function of individual nucleotides within previously known enhancers ${ }^{16-19}$ as well as scan genomic sequence for enhancer activity ${ }^{20}$. These methods have either used UTR tags to assay from thousands to hundreds of thousands of fragments in parallel ${ }^{16-19}$ or have had to confine the potential enhancer itself to the UTR in order to assay genome-scale complexities ${ }^{20}$. Here we use a novel variation on these high-throughput enhancer screening methods to identify regions of the Drosophila genome with increased activity under hypoxia. Our technique combines randomly sheared genomic fragments to be assayed for activity with a UTR randomer tag system for highly multiplexed tracking of transcriptional activity. The construct library is modularly synthesized in vitro making the relative placement of construct elements easily mutable. This is in contrast to a similar method called STARR-Seq ${ }^{20}$ that requires the potential enhancer itself be placed 
74 75 76

downstream of the transcription start site. Although enhancers are known to function at variable distance and orientation with respect to a target promoter $^{21,22}$ their strength has been shown to be modulated by their position relative to the target promoter $^{23}$ and transcriptional read-through has been shown to attenuate their activity ${ }^{24}$. The method in this paper allows the regulatory element to be placed at the discretion of the experimenter. Additionally, the previously published library construction methods ${ }^{16-20}$ require microbial propagation of DNA libraries whereas we present a simpler entirely in vitro strategy. The work presented here is the first implementation of a massively parallel reporter assay to study cis-regulatory activity during an environmental stress response. A library of 4,599,881 random 400-500 bp fragments spanning the Drosophila melanogaster genome was used to identify 31 hypoxic enhancer regions. The regions coincide with genes up-regulated under hypoxia and with binding site motifs from multiple transcription factors involved in the hypoxic response. This work provides mechanistic details of the hypoxic response by empirically identifying regulatory regions that drive hypoxic transcription, linking them to target genes from RNAseq differential expression data, and identifying trans-acting factors in silico. Investigating the hypoxic response in Drosophila allows us to corroborate previous work on hypoxic gene regulation ${ }^{7,9,10}$ and a previous empirical genome-wide enhancer screen $^{20}$. This genome-wide scan demonstrates the complexity of the hypoxic response, which involves multiple regulators acting in concert to control the expression of a wide variety of targets.

\section{MATERIALS AND METHODS}

All DNA sequencing was performed on the Illumina HiSeq. All PCR reactions contained a final concentration of 400nM of each primer and used Phusion Polymerase in 1X HF buffer. All oligonucleotide sequences are listed in Supplementary File S1.

\section{Library synthesis}

The linear reporter library used to assay enhancer activity was constructed entirely in vitro (Figure 1A). The sequence space being assayed for enhancer activity, in this case the Drosophila melanogaster genome, was sonically sheared to generate random enhancer-sized fragments. 
105 Adapter ligation and 5' PCR addition were used to add the Illumina first-end sequence upstream

106 of the sheared DNA and part of the minimal promoter downstream. 5' PCR additions are used to

107 add minimal promoter elements, an intron to stabilize mRNAs ${ }^{25}$, the $20 \mathrm{~N}$ randomer tag, and

108 Illumina paired-end sequence upstream of an arbitrary ORF, in this case GFP. The synthetic

109 minimal promoter used was designed to contain several core motifs and has been shown to

110 function with a wide range of enhancers ${ }^{26}$. The two fragments are then ligated together to create

111 the final construct library pictured in Figure 1A. The reporter library was diluted to a target of

$11210,000,000$ molecules and regenerated by PCR so that the library could be adequately

113 characterized by paired-end sequencing. An aliquot of the reporter library is used for paired-end

114 sequencing to match randomer tags located in the 5' UTR to the non-transcribed genomic region

115 driving their expression. The library is then transfected into cells for massively parallel enhancer

116 assay (Figure 1B).

117

118 Drosophila melanogaster strain Oregon-R genomic DNA was sonically sheared using the

119 BioRuptor. 400-500bp fragments were isolated by gel electrophoresis then end-repaired using

120 Blunt Enzyme mix (NEB) and 3' adenylated using Klenow exo- (NEB). This sample was then

121 ligated to an asymmetric adapter with T-overhang composed of annealed oligonucleotides

122 Genomic-Adapter-1 and Genomic-Adapter-2. The ligation product was gel-purified and used as

123 PCR template with primers Illumina P5 and Genomic-R to create a library of molecules

124 containing a random 400-500 bp stretch of Drosophila melanogaster genomic sequence between

125 the Illumina end one sequence and the beginning of a synthetic promoter. Separately, The GFP

126 coding sequence followed by the SV40 terminator was PCR amplified from plasmid pGreen-H-

127 Pelican with primers GFP-F and SV40-R. This product was then used as template for a PCR

128 reaction using primers SV40-R and Marker-1-F. This product was then used as template for a

129 PCR reaction using primers SV40-R and Marker-2-F. This product was then used as template for

130 a PCR reaction using primers SV40-R and Marker-3-F to create a library of molecules

131 containing a GFP sequence downstream of a minimal promoter with randomer tag and Illumina

132 paired-end sequences. The genomic sequence-containing library and minimal promoter library

133 were then 3' adenylated and 3' thymidylated respectively with Klenow exo- then ligated

134 together. The heterodimer (1819-1919 bp) was gel-purified and subsequently selected for proper

135 orientation by PCR with primers SV40-R and Illumina P5. To reduce library complexity to a 
136 scale that was tractable by paired-end sequencing, DNA was quantified using the Qubit system

137 (Invitrogen) and serially diluted to produce an estimated 10,000,000 molecules that were used as 138 template to regenerate the library by PCR with primers SV40-R and Illumina P5. An aliquot of

139 this library was used as template for a PCR reaction with primers Illumina-P7 and Illumina-P5 to 140 generate a paired-end Illumina-sequencing library such that the first-end sequence contained the 141 beginning of the genomic region and the paired-end sequence contained the corresponding 142 randomer tag (Figure 1A). Aliquots were also used to generate transfectable quantities of the 143 full-length reporter library by PCR amplification of the entire fragment using primers SV40-R 144 and Illumina-P5. The final construct library sequence is available in the supplementary material 145 (Supplementary File S2).

Transfection, RNA extraction, and randomer tag sequencing

Six $5 \mathrm{~mL}$ flasks were plated to $80 \%$ confluency with S2 cells (contributed by Ken Prehoda lab, 150 University of Oregon) and transfected with Fugene HD and 2.6ug reporter library DNA at a 3:1 ratio. The following day three plates were placed under hypoxia $(99.5 \% \mathrm{~N} 2$ and $0.5 \% \mathrm{O} 2)$ for five hours and thirty minutes and three were left in atmospheric conditions. Total RNA from both conditions was extracted using Trizol and treated with DNAse Turbo (Ambion). RNA was converted to cDNA with SuperScipt III first strand synthesis kit (Invitrogen) using oligo dT20 primers. cDNA was used as template for PCR with primers flanking the randomer tag to create an amplicon ready for Illumina sequencing. All PCR reactions used Illumina-P7 reverse primer and the following barcoded forward primers to allow multiplexing: RNA-BC-1 for hypoxic sample 1, RNA-BC-2 for hypoxic sample 2, RNA-BC-3 for hypoxic sample 3, RNA-BC-4 for normoxic sample 1, RNA-BC-5 for normoxic sample 5, RNA-BC-6 for normoxic sample 6 . The resulting 178-bp amplicons were combined and sequenced on the Illumina Hiseq.

\section{RNAseq}

RNA from the same experiments used to quantify enhancer activity was used for RNAseq. mRNA was purified using Dynabeads (Invitrogen) from 10ug of total RNA and chemically 166 fragmented using Ambion Fragmentation Reagent. cDNA libraries were made with SuperScipt 
167 III first strand synthesis kit using random hexamer primers followed by second-strand synthesis

168 with DNA Pol I (NEB). The double stranded DNA was end-repaired using NEB Quick Blunting

169 Kit and 3' adenylated using Klenow exo-. The samples were ligated to divergent Illumina

170 adapters with in-line barcodes (Hypoxic GGTTC, Normoxic CTTCC) and PCR amplified with

171 Illumina primers. 300-450 bp fragments were gel-purified and sequenced on the Illumina HiSeq

172 (hypoxic condition: Accession SRX467593, normoxic condition: Accession SRX467591).

$1736,855,528$ reads from each sample were aligned to the Drosophila melanogaster transcriptome

174 (Flybase, r5.22) using TopHat ${ }^{27}$. The bam outputs were analyzed by cufflinks and the resulting

175 transcripts.gtf files were compared using cuffdiff to identify differentially expressed genes

176 (Supplementary File S3). Some ncRNAs were also analyzed for differential expression. As they

177 are not present in the transcriptome build, RNAseq reads were aligned to each ncRNA using

178 Bowtie $2^{28}$ and their expression level is reported by normalized number of aligned reads in each

179 condition.

180

181

\section{Computational enhancer activity analysis pipeline}

182

183

All scripts and a tutorial are available in Supplementary File S4. Paired-end fastq files

184

(Accession SRX468157) linking genomic regions in the first-end read to randomer tags in the

185

paired-end read were parsed to a fasta file with the randomer tag as the sequence name and the

186

genomic sequence as the sequence. This file containing 32,061,029 sequences was aligned to

187

the Drosophila melanogaster genome (dm3) using Bowtie $2^{28}$. Reads were processed into a

188

match-list linking randomer tags to the genomic coordinates of their corresponding test

189 sequence.

190

191

Randomer tags from hypoxic and normoxic RNA amplicon sequencing were extracted from

192

fastq files (Accessions SRX468694, SRX468097) and experimental replicates were separated by

193

barcode. 18,261,667 randomer tags from hypoxic sample 1, 14,226,458 from hypoxic sample 2,

194

14,697,154 from hypoxic sample 3, 14,406,854 from normoxic sample 1, 14,988,132 from

195

normoxic sample 2, and 11,516,478 from normoxic sample 3 were referenced to the paired-end

196 match list to generate genome-wide enhancer activity tables by $100 \mathrm{bp}$ bins. The genomic

197 fragments ranged from $400-500 \mathrm{bp}$ so the bin corresponding to the alignment as well as the four 
198 downstream bins were credited 1 count. In the cases where randomer tags linked to multiple

199 genomic fragments, bins were credited a fraction of a count based on the likelihood of that

200 linkage in the paired-end match data. This created a count table of enhancer activity in each

201 replicate at each $100 \mathrm{bp}$ region of the Drosophila melanogaster genome.

202

203 The count table was then analyzed for differential activity between hypoxic and normoxic

204 replicates using a negative binomial test in the DESeq ${ }^{29}$ package within the R programming

205 language. The bins were filtered by overall count $(\theta=0.5)$ and the test was run with default

206 variance estimation. This generated a p-value and a p-value adjusted for multiple hypothesis

207 testing (Benjamini-Hochberg procedure) for each 100bp bin. Hypoxic enhancer regions were

208 defined at bins up-regulated under hypoxia with adjusted p-value $<0.1$ (p-value $<1.55$ e-05) and

209 extended to include adjacent bins with p-value $<0.05$.

210

211 In order to compare our results to those of STARR-seq ${ }^{20}$, we also identified statistically

212 significant S2 baseline enhancers within the normoxic replicates. In this case, the negative

213 binomial test was performed between counts in the transfected DNA library and counts in the

214 RNA-sequenced barcodes for each $100 \mathrm{bp}$ bin across the Drosophila melanogaster genome in

215 order to identify genomic regions enriched for baseline enhancer activity. Peaks were identified

216 with an adjusted p-value $<0.018$ (Benjamini-Hochberg procedure) and enhancers were defined

217 as the $500 \mathrm{bp}$ interval surrounding the activity peak in order to maintain consistency with

218 STARR-seq data.

219

220

Enhancer sequence motif analysis

221

222

Identified hypoxic enhancer regions were searched for stress transcription factor binding sites

223 using the BoBro BBS motif-scanning algorithm ${ }^{30}$ with position weight matrices from the JASPAR database ${ }^{31}$. This algorithm was used to identify binding site positions and calculate a global p-value of enrichment for HIF-1 (JASPAR ID: MA0259.1), FOXO (MA0480.1), HSF

226 (MA0486.1) and NF-kB (MA0105.3) binding sites in enhancer sequences compared to the Drosophila melanogaster genome background. 
229

230

231

232

233

234

235

236

237

238

239

240

241

242

243

244

245

246

247

248

249

250

251

252

253

254

255

256

257

258

259

\section{RESULTS}

\section{Discovered hypoxic enhancers}

Transcriptional activity from 4,599,881 fragments that were 400-500bp in size, spanning the Drosophila melanogaster genome at $17.39 \mathrm{X}$ coverage, was analyzed by $100 \mathrm{bp}$ bins and 31 significant hypoxic enhancer regions (q-value $<0.1$, p-value $<1.55$ e-05) were identified (Table 1, Supplementary File S5). These enhancer regions range in size from 100 to $800 \mathrm{bp}$ and confer 2 to 18-fold changes in expression under hypoxia. The discovered enhancers are found throughout the genome and are located proximally to genes up-regulated under hypoxia in our RNAseq experiments. The ten most strongly up-regulated genes all contain a discovered enhancer within $20 \mathrm{~kb}$. 16 of 31 discovered enhancers are located within $20 \mathrm{~kb}$ of one of the $90 \mathrm{up}$-regulated genes. The probability of this positional overlap occurring by chance is 1.43 e-14 using an exact binomial test, supporting that the discovered enhancers are linked to endogenous gene expression and implicating their likely targets. 4 additional enhancers are proximal to genes previously observed to be up-regulated under hypoxia in Drosophila ${ }^{32}$.

\section{Location of hypoxic enhancers}

Of the 20 hypoxic enhancer regions proximal (within 20kb) to hypoxic up-regulated genes, 6 fall in the promoter region of the putative target gene (Figure 2, Table 1). All six of these are the homologous Hsp70B enhancers. Six enhancers were found in introns of putative target genes (Table 1). These intronic enhancers may be placed proximal to alternate transcription start sites in order to confer isoform specific up-regulation as seen in the case of Sima, the Drosophila HIF-1 $\alpha$ homologue (Figure 3). Two enhancers were found in introns of genes neighbouring the putative target and one was found in the ORF of the putative target. The remaining five were found in intergenic space up or downstream of putative target genes, as seen for the enhancer region $13 \mathrm{~kb}$ downstream of the transcriptional regulator hairy (Figure 4). Interestingly, three of the five intergenic enhancers were located immediately proximal to a ncRNA. All of these ncRNAs were themselves up-regulated under hypoxia (Table 2). 
260

261

262

263

264

265

266

267

268

269

270

271

272

273

274

275

276

277

278

279

280

281

282

283

284

285

286

287

288

289

\section{Transcription factor binding motifs}

Identified enhancer regions are enriched for binding sites of stress response transcription factors involved in hypoxia. Transcription factors HSF, HIF-1, FOXO, and NF-kB showed highly significant global enrichment across the enhancer regions (Table 3). Binding sites occurring in each individual enhancer are listed in Table 1. 26 of 31 enhancer regions contain binding motifs for at least one of these transcription factors and many contain binding sites for several. In addition to a pair of HSF binding sites, The Hsp70B promoter proximal enhancers contain binding sites for FOXO and HIF-1 (Figure 2). The intronic Sima enhancer (Figure 3) contains a pair of HIF-1 binding sites, possibly allowing autoregulation, and also contains a NF-kB binding site. The enhancer region downstream of hairy contains HSF, FOXO, and HIF-1 binding sites (Figure 4).

\section{Overlap with STARR-seq enhancers}

Our data correlate strongly with a previous genome-wide empirical assay of Drosophila transcriptional enhancers. STARR-seq ${ }^{20}$ was used to identify 5499 enhancers operating in S2 cells under normal conditions. These enhancers were defined as $500 \mathrm{bp}$ intervals surrounding statistically significant peaks in enhancer activity (Adjusted p-value $<0.018, \mathrm{p}<0.001$ ). In order to generate a similar dataset for comparison, we identified genomic regions showing significant enrichment in normoxic S2 cells. Similar to STARR-seq, we defined enhancers as $500 \mathrm{bp}$ intervals surrounding peaks with an adjusted p-value less than 0.018 (unadjusted $<$ 0.00043). This yielded a list of 1007 baseline S2 enhancers (Supplementary File S6). 466 of these (46.3\%) overlap the enhancers identified using STARR-seq. The probability of a $500 \mathrm{bp}$ fragment overlapping the STARR-seq set by chance is 0.0462 . An exact binomial test (463 hits, 1007 trials, 0.0462 background probability) generates a p-value of 0 for the overlap between our data and STARR-seq. The ratio of overlap is higher when only the most enriched peaks from out dataset are examined. 19 of the 25 most enriched enhancer peaks $(76 \%$, p-value $=5.66 \mathrm{e}-21)$ overlap with the STARR-seq dataset. This high degree of overlap demonstrates a robust ability to have identified active enhancers. 
290

291

292

293

294

295

296

297

298

299

300

301

302

303

304

305

306

307

308

309

310

311

312

313

314

315

316

317

318

319

320

\section{DISCUSSION}

We used a novel parallelized reporter assay to conduct the first genome-wide functional enhancer screen of a cellular response to environmental stress. Our work demonstrates a new method with wide applicability and identifies DNA regulatory sequences conferring hypoxic activity. We identify 31 hypoxic enhancer regions and analyze them with respect to up-regulated hypoxic genes and stress response transcription factors.

RNA-Seq was performed on the same RNA pools used to quantify hypoxic enhancer activity in order to identify putative target genes proximal to identified enhancer regions. Differentially expressed genes identified in our RNA-Seq experiments are corroborated by previous analyses of the Drosophila hypoxic response $\mathrm{e}^{32,33}$. The majority of enhancer regions were proximal (within $20 \mathrm{~kb}$ ) to endogenously up-regulated genes, indicating that our enhancer assay identifies active in vivo regulatory elements. We identified enhancer regions proximal to previously described hypoxic genes including lactate dehydrogenase ${ }^{6,32}$, the transcriptional regulator hairy ${ }^{34}$, the reductase $\mathrm{Wwox}^{35}$, and the cell cycle inhibitor scyl ${ }^{36}$. Additionally, the Hsp70B promoter proximal enhancers identified in our assay have been previously shown to be active in vivo ${ }^{37,38}$. The large positional overlap between up-regulated genes and enhancer regions allowed analysis of the architecture of hypoxic regulation. Interestingly, while some enhancers were found at the promoters of putative target genes, the majority of enhancer regions were found in introns and intergenic space. Enhancers were found in introns of putative target genes as well as introns of neighboring genes (Table 1). Enhancer regions in intergenic space corresponded with known ncRNA loci and in each case the ncRNA was itself up-regulated under hypoxia (Table 2). These findings highlight the unbiased view of the regulatory landscape provided by genome-wide empirical assays and underscore the prevalence of activity outside of promoter regions.

Some of the enhancer regions were not proximal to an identifiable up-regulated gene. These enhancers could act on more distal targets, on proximal targets with expression too low to be detected by our RNA-Seq experiment, or they may have activity in isolation but be attenuated by other elements in their native hypoxic context. Conversely, many up-regulated genes did not have a proximal enhancer identified by our screen. This could be due to a requirement of action 
321 from multiple disjunct regulatory modules at the native locus or lack of resolution in our assay.

322 The resolution of our assay was attenuated by the coincidence of randomer tags with multiple

323 genomic regions. Other randomer tag-based approaches test orders of magnitude fewer

324 fragments and hence largely avoid barcode collision ${ }^{16-19}$. This problem is circumvented in

325 STARR-Seq ${ }^{20}$ by confining placement of the potential enhancer to the transcribed region so that

326 it can be assayed directly by RNA sequencing. Future uses of our technique will benefit from

327 further optimization of library synthesis to allow a greater number of randomer tags into the

328 library. Nonetheless, the technique is highly functional in its present state and introduces a

329 simpler and more versatile library synthesis approach. Indeed, our data show a large degree of

330 overlap with STARR-Seq with respect to baseline transcriptional enhancers in Drosophila S2

331 cells. Furthermore, this work presents a large list of empirically identified hypoxia-induced

332 enhancer regions robust to false discovery rate that coincide with the most highly up-regulated

333 hypoxic genes.

334

335 The transcription factors HIF-1, HSF, NF-kB , and FOXO regulate hypoxic gene expression and

336 have been shown to exhibit overlapping activity and reciprocal regulation ${ }^{9-11,39,40}$. The enhancer

337 regions identified in this study are highly enriched for their binding site motifs and many display

338 multiple sites allowing signal integration of stress response pathways. We observe an intronic

339 enhancer in Sima which contains both HIF-1 and NF-kB binding sites, suggesting HIF-1

340 autoregulation and integration of NF-kB signaling at a basal level in the hypoxic response. The

341 enhancer region, while intronic to the full-length Sima transcript isoforms, is upstream of an

342 alternative transcriptional start site that produces a transcript isoform that is up-regulated after

343 hypoxia, whereas the full-length isoforms do not have altered expression after hypoxic stress.

344 This short isoform lacks the bHLH and PAS domains of the full-length isoform, suggesting it

345 neither binds DNA nor heterodimerizes. Interestingly, this hypoxic regulation of a short isoform

346 resembles the hypoxic induction of a short isoform of the HIF-1 regulator fatiga (Drosophila

347 HIF-1 Prolyl Hydroxylase) by an intronic HIF-1 enhancer ${ }^{41}$.

348

349 Our findings reiterate the complexities of the hypoxic response while providing new details. The

350 enhancer regions identified demonstrate regulatory activity distributed throughout non-coding

351 genomic space and underscore the role of intronic enhancers in the hypoxic response. We 
352 observe coincidence between enhancer regions and ncRNA activity in agreement with previous

353 evidence showing local transcription to be a general property of active enhancers ${ }^{42}$. We present 354 a set of sequences capable of driving hypoxia-specific expression and demonstrate a new

355 genome-wide technique for the identification of context-specific enhancers.

356

357 ACKNOWLEDGEMENT

358 We thank Paul Etter and Doug Turnbull for advice on Illumina library preparation.

359

360

361 
362

363

364

365

366

367

368

369

370

371

372

373

374

375

376

377

378

379

380

381

382

383

384

385

386

387

388

389

390

391

392

\section{REFERENCES}

1. Bulger,M. and Groudine,M. (2011) Functional and mechanistic diversity of distal transcription enhancers. Cell, 44, 327-339.

2. Perry,M.W., Boettiger,A.N. and Levine,M. (2011) Multiple enhancers ensure precision of gap gene-expression patterns in the Drosophila embryo. Proc. Natl. Acad. Sci. USA, 108, 1357013575.

3. Lagha,M., Bothma,J.P. and Levine,M. (2012) Mechanisms of transcriptional precision in animal development. Trends Genet., 28, 409-416.
4. Arnosti,D.N. and Kulkarni,M.M. (2005) Transcriptional enhancers: Intelligent enhanceosomes or flexible billboards?. J. Cell. Biochem., 94, 890-898.

5. Swanson,C., Schwimmer,D.B. and Barolo,S. (2011) Rapid evolutionary rewiring of a structurally constrained eye enhancer. Curr. Biol., 21, 1186-1196.

6. Bruick,R.K. and McKnight,S.L. (2001) A conserved family of prolyl-4-hydroxylases that modify HIF. Sci. Signal., 294, 1337-1340.

7. Lavista-Llanos,S., Centanin,L., Irisarri,M., Russo,D.M., Gleadle,J.M., Bocca,S.N., Muzzopappa,M., Ratcliffe,P.J. and Wappner,P. (2002) Control of the hypoxic response in Drosophila melanogaster by the basic helix-loop-helix PAS protein similar. Mol. Cell Biol., 22, 6842-6853.

8. Wang,G.L. and Semenza,G.L. (1993) General involvement of hypoxia-inducible factor 1 in transcriptional response to hypoxia. Proc. Natl. Acad. Sci. USA, 90, 4304-4308.

9. Rius,J., Guma,M., Schachtrup,C., Akassoglou,K., Zinkernagel,A.S., Nizet,V., Johnson,R.S., Haddad,G.G. and Karin,M. (2008) NF-kappaB links innate immunity to the hypoxic response through transcriptional regulation of HIF-1alpha. Nature, 453, 807-811.

10. van Uden,P., Kenneth,N.S., Webster,R., Müller,H.A., Mudie,S, and Rocha,S. (2011)

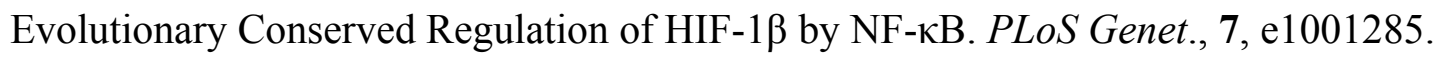

11. Scortegagna,M., Cataisson,C., Martin,R.J., Hicklin,D.J., Schreiber,R.D., Yuspa,S.H. and Arbeit,J.M. (2008) HIF-1 $\alpha$ regulates epithelial inflammation by cell autonomous NFkB activation and paracrine stromal remodeling. Blood, 111, 3343-3354.

12. Metzker,M.L. (2010) Sequencing technologies - the next generation. Nat. Rev. Genet., 11, $31-46$. 
393

394

395

396

397

398

399

400

401

402

403

404

405

406

407

408

409

410

411

412

413

414

415

416

417

418

419

420

421

13. Wang,Z., Gerstein,M. and Snyder,M. (2009) RNA-Seq: a revolutionary tool for transcriptomics. Nat. Rev. Genet., 10, 57-63.

14. Johnson,D., Mortazavi,A., Myers,R.M. and Wold,B. (2007) Genome-wide mapping of in vivo protein-DNA interactions. Science, 316, 1497-1502.

15. Song,L., Zhang,Z., Grasfeder,L.L., Boyle,A.P., Giresi,P.G., Lee,B.K., Sheffield,N.C., Graf,S., Huss,M., Keefe,D. et al. (2011) Open chromatin defined by DNaseI and FAIRE identifies regulatory elements that shape cell-type identity. Genome research, 21,1757-1767.

16. Kwasnieski,J.C., Mogno,I., Myers,C.A., Corbo,J.C. and Cohen,B.A. (2012) Complex effects of nucleotide variants in a mammalian cis-regulatory element. Proc. Natl. Acad. Sci. USA, 109, 19498-19503.

17. Patwardhan,R.P., Hiatt,J.B., Witten,D.M., Kim,M.J., Smith,R.P., May,D. Lee,C., Andrie,J.M., Lee,S., Cooper,G.M., Ahituv,N., Pennacchio,L.A. and Shendure,J. (2012) Massively parallel functional dissection of mammalian enhancers in vivo. Nat. Biotechnol., 30, 265-270.

18. Melnikov A, Murugan A, Zhang X, Tesileanu T, Wang L, Rogov,P., Feizi,S., Gnirke,.A, Callan,C.G., Kinney,J.B., Kellis,M., Lander,E.S. and Mikkelsen,T.S. (2012) Systematic dissection and optimization of inducible enhancers in human cells using a massively parallel reporter assay. Nat. Biotechnol., 30, 271-277.

19. Kheradpour P, Ernst J, Melnikov A, Rogov P, Wang L, Zhang,X., Alston,J., Mikkelsen,T.S. and Kellis,M. (2013) Systematic dissection of regulatory motifs in 2000 predicted human enhancers using a massively parallel reporter assay. Genome Res., 23, 800-811.

20. Arnold,C.D., Gerlach,D., Stelzer,C., Boryń,Ł.M., Rath,M. and Stark,A. (2013) GenomeWide Quantitative Enhancer Activity Maps Identified by STARR-seq. Science, 339, 1074-1077. 21. Bulger,M. and Groudine,M. (2011) Functional and mechanistic diversity of distal transcription enhancers. Cell, 44, 327-339.

22. Banerji,J., Rusconi.,S and Schaffner,W. (1981) Expression of a $\beta$-globin gene is enhanced by remote SV40 DNA sequences. Cell, 27, 299-308.

23. Amit,R., Garcia,H.G., Phillips,R. and Fraser,S.E. (2011) Building enhancers from the ground up: a synthetic biology approach. Cell, 146, 105-118. 
422

423

424

425

426

427

428

429

430

431

432

433

434

435

436

437

438

439

440

441

442

443

444

445

446

447

448

449

450

451

24. Erokhin,M., Davydova,A., Parshikov,A., Studitsky,V.M., Georgiev,P. and Chetverina,D. (2013) Transcription through enhancers suppresses their activity in Drosophila. Epigenetics Chromatin, 6, 31 .

25. Zieler,H. and Huynh,C.Q. (2002) Intron-dependent stimulation of marker gene expression in cultured insect cells. Insect Mol. Biol., 11, 87-95.

26. Pfeiffer,B.D., Jenett,A., Hammonds,A.S., Ngo,T.T.B., Misra,S., Murphy,C., Scully,A., Carlson,J.W., Wan,K.H., Laverty,T.R., Mungall,C., Svirskas,R., Kadonaga,J.T., Doe,C.Q., Eisen,M.B.,

Celniker,S.E. and Rubin,G.M. (2008) Tools for neuroanatomy and neurogenetics in Drosophila. Proc. Natl. Acad. Sci. USA, 105, 9715-9720.

27. Trapnell,C., Lior,P. and Salzberg,S.L. (2009) TopHat: discovering splice junctions with RNA-Seq. Bioinformatics, 25, 1105-1111.

28. Langmead,B. and Salzberg,S.L. (2012) Fast gapped-read alignment with Bowtie 2. Nat. Methods, 9, 357-359.

29. Anders,S. and Huber,W. (2010) Differential expression analysis for sequence count data. Genome Biol., 11, R106.

30. Ma,Q., Zhang,H., Mao,X., Zhou,C., Liu,B., Chen,X., and Xu,Y. (2014) DMINDA: an integrated web server for DNA motif identification and analyses. Nucleic Acids Res., gku315.

31. Mathelier,A., Zhao,X., Zhang,A.W., Parcy,F., Worsley-Hunt,R., Arenillas,D.J., Buchman,S., Chen,C., Chou,A., Ienasescu,H., Lim,J., Shyr,C., Tan,G., Zhou,M., Lenhard,B., Sandelin,A. and Wasserman,W.W. (2013) "JASPAR 2014: an extensively expanded and updated open-access database of transcription factor binding profiles." Nucleic Acids Res, gkt997.

32. Li,Y., Padmanabha,D., Gentile,L.B., Dumur,C.I., Beckstead,R.B. and Baker,K.D. (2013) HIF-and Non-HIF-Regulated Hypoxic Responses Require the Estrogen-Related Receptor in Drosophila melanogaster. PLoS Genet, 9, e1003230.

33. Liu,G., Roy,J. and Johnson,E.A. (2006) Identification and function of hypoxia-response genes in Drosophila melanogaster. Physiol. Genomics, 25, 134-141.

34. Zhou,D., Xue,J., Lai,J.C., Schork,N.J., White,K.P. and Haddad,G.G. (2008) Mechanisms underlying hypoxia tolerance in Drosophila melanogaster: hairy as a metabolic switch. PLoS Genet., 4, e1000221. 
452

453

454

455

456

457

458

459

460

461

462

463

464

465

466

467

468

469

470

471

472

35. O'Keefe,L.V., Colella,A., Dayan,S., Chen,Q., Choo,A., Jacob,R., Price,G., Venter,D., and Richards,R.I. (2011) Drosophila orthologue of WWOX, the chromosomal fragile site FRA16D tumour suppressor gene, functions in aerobic metabolism and regulates reactive oxygen species. Hum. Mol. Genet., 20, 497-509.

36. Scuderi,A., Simin,K., Kazuko,S.G., Metherall,J.E. and Letsou,A. (2006) scylla and charybde, homologues of the human apoptotic gene RTP801, are required for head involution in Drosophila. Dev Biol, 291, 110-122.

37. Tian,S., Haney,R. and Feder,M. (2010) Phylogeny disambiguates the evolution of heatshock cis-regulatory elements in Drosophila. PLoS One, 5, e10669.

38. Li,D., Li,G., Wang,K., Liu,X., Li,W., Chen,X. and Wang,Y. (2012) Isolation and functional analysis of the promoter of the amphioxus Hsp70a. Gene, 510, 39-46.

39. Hsu,A.L., Murphy,C.T. and Kenyon,C. (2003) Regulation of aging and age-related disease by DAF-16 and heat-shock factor. Science, 300, 1142-1145.

40. Wang,M.C., Bohmann,D. and Jasper,H. (2005) JNK extends life span and limits growth by antagonizing cellular and organism-wide responses to insulin signaling. Cell, 12, 115-125.

41. Acevedo,J.M., Centanin,L., Dekanty,A. and Wappner,P. (2010) Oxygen Sensing in Drosophila: Multiple Isoforms of the Prolyl Hydroxylase Fatiga Have Different Capacity to Regulate HIFa/Sima. PLoS One, 5, e12390.

42. Andersson,R., Gebhard,C., Miguel-Escalada,I., Hoof,I., Bornholdt,J., Boyd,M., Chen,Y., Zhao,X., Schmidl,C., Suzuki,T., et al. (2014) An atlas of active enhancers across human cell types and tissues. Nature, 507, 455-461. 


\section{Figure 1 (on next page)}

\section{Library Synthesis}

(A) The enhancer library is synthesized entirely in vitro. DNA of interest is fragmented (step 1) and ligated to divergent adapters (step 2) leaving potential enhancer fragments with Illumina sequence on one side and the beginning of the synthetic minimal promoter on the other. The GFP gene is used as a template for a series of 5' PCR additions in order to add Illumina sequence, $20 \mathrm{~N}$ randomer tag, and the majority of the minimal promoter and intron (step 3). The two sides are ligated together to create a linear construct with complexity in the enhancer region upstream of the transcription start site as well as complexity in the randomer tag region in the 5' UTR (step 4). The sample is submitted to paired-end sequencing in order to match the potential enhancer region to the randomer tag in the 5' UTR that is used to report its activity. (B) The enhancer library is transfected into cells (step 1) and total RNA is purified and reverse transcribed to create cDNA (step 2). The cDNA is used as template for a PCR reaction (step 3) with a reverse primer complimentary to the Illumina end 2 sequence present in the construct and a forward primer complimentary to the stretch of the minimal promoter upstream of the randomer tag. The forward primer adds Illumina end 1 seqeunce and an experimental barcode for multiplexing. This amplicon is ready to be loaded onto the Illumina flow cell for single-end sequencing of randomer tags (step 4) in order to quantify enhancer activity. 
A DNA space to be querigd for enhancer activity, in this case the Drosophila genome, is ranacm yy fragmented and ligated to divergent adapters to prepare test fragments for enhancer assay

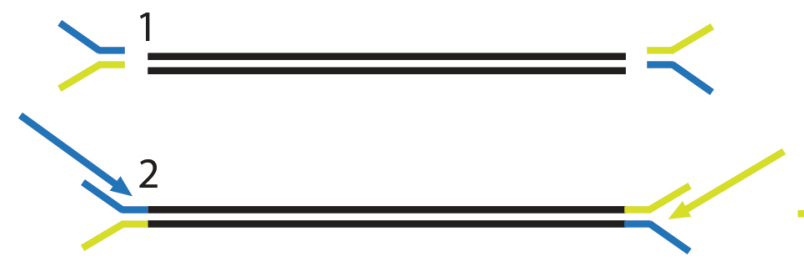

Illumina priming sequence, $20 \mathrm{~N}$ randomer tag, and Manumifinimationnoterareadeled to the GFP ORF via successive PCR additions

\section{4}

The two pools are ligated together generating a linear construct library containing complexity in both the enhancer and randomer tag positions

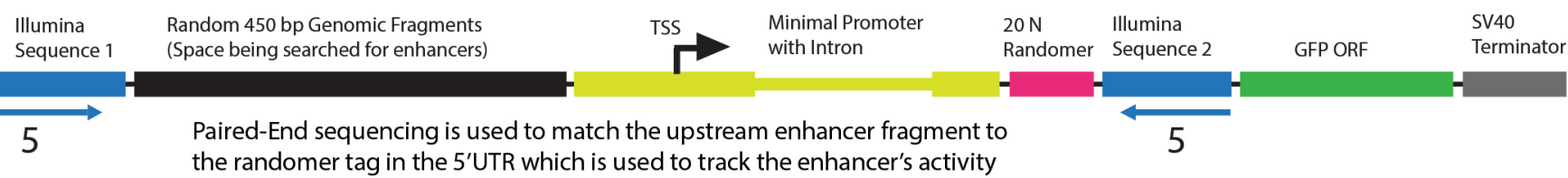

A
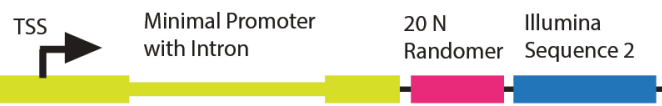

Construct library is transfected

1
ected
erest

into cells of intere

\section{Cells}

mRNA is purified and

2 reverse transcribed

B

Randomers tags are Illumina-sequenced counted, and mapped to paired end matching data (fig1 $\mathrm{A}$ ) in order to quantify enhancer activity Peer) reviewing PDF | (2015:09:6791:1:0:REVIEW 9 Nov 2015)

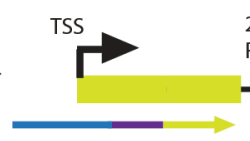

$20 \mathrm{~N}$

Randomer Sequence 2
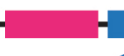

Selective primers prepare construct

3 derived RNAs for Illumina sequencing

$20 \mathrm{~N} \quad$ Illumina

Randomer Sequence 2 
Figure 2 (on next page)

Hypoxic Enhancer Activity by 100bp Bins at the Hsp70B Locus

Each open circle plots the $p$-value of the difference in randomer tag counts mapping to that $100 \mathrm{bp}$ bin between normoxia and hypoxia. Green bars show enhancer regions discovered by our genome-wide screen. (A) The four Hsp70B homologues highlighted in pink are all upregulated under hypoxia and contain homologous promoter proximal hypoxic enhancer regions. Additionally, a fifth homologous enhancer region lacking an ORF was discovered at the locus. (B) The close up of the Hsp70Ba enhancer region shows the position of multiple stress response transcription factor binding sites. 


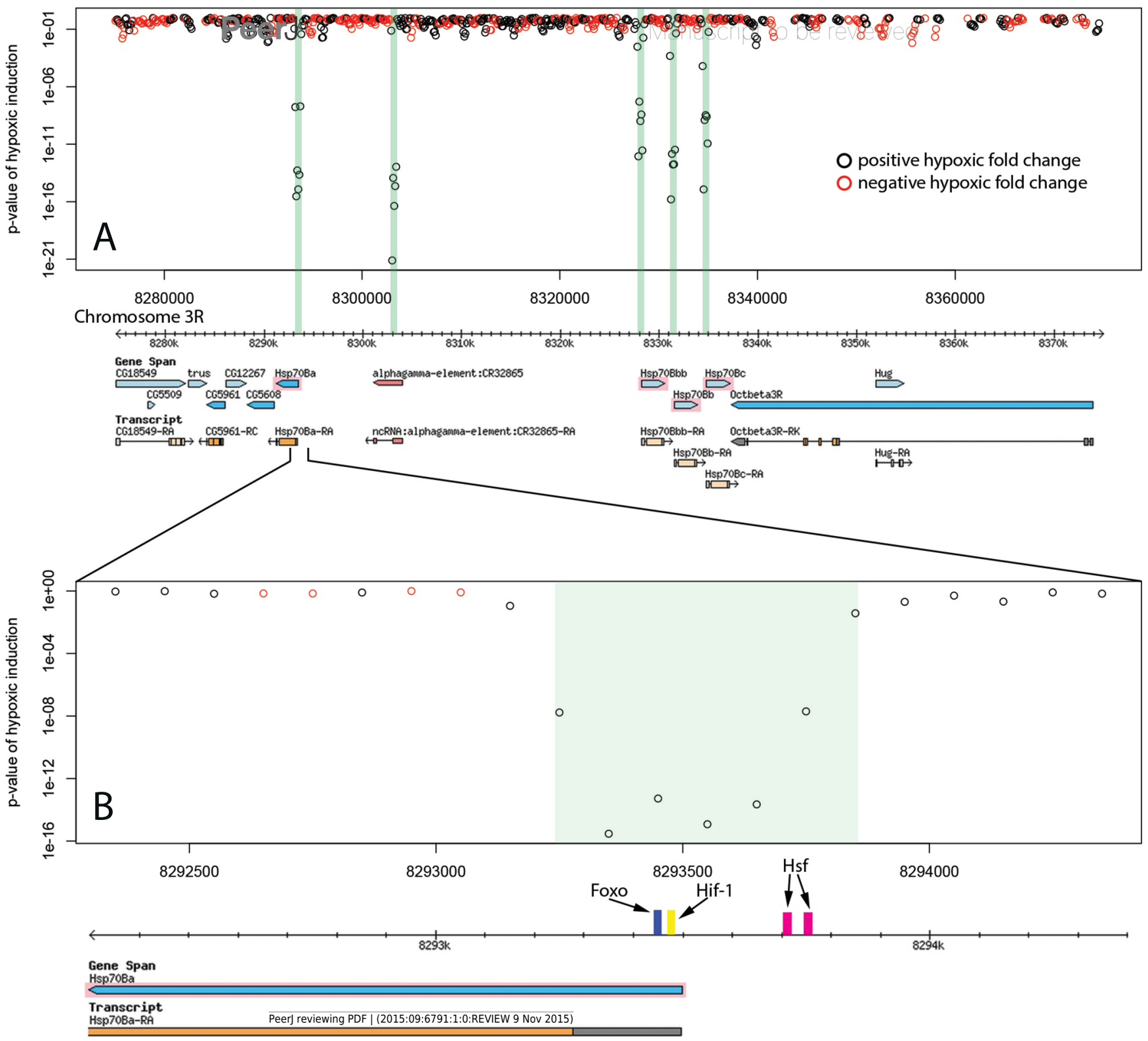


Figure 3 (on next page)

Hypoxic Enhancer Activity by 100 bp Bins at the Sima (HIF-1 $\alpha$ ) Locus

Each open circle plots the $p$-value of the difference in randomer tag counts mapping to that $100 \mathrm{bp}$ bin between normoxia and hypoxia. The green bar shows the enhancer region discovered by our genome-wide screen. (A) HIF-1 is the master hypoxic regulator and is itself regulated transcriptionally under hypoxia. Our RNASeq data shows hypoxia induces upregulation of the isoform highlighted in pink. We identify an intronic hypoxic enhancer upstream of the transcription start site of this isoform. (B) The close up of the Sima intronic enhancer region shows both HIF-1 and NF-kB binding sites. 
Figure 4(on next page)

Hypoxic Enhancer Activity by 100bp Bins at the hairy Locus

Each open circle plots the $p$-value of the difference in randomer tag counts mapping to that $100 \mathrm{bp}$ bin between normoxia and hypoxia. The green bar shows the enhancer region discovered by our genome-wide screen. (A) The hairy gene produces a negative transcriptional regulator that is up-regulated during hypoxia. We identify an active hypoxic enhancer $13 \mathrm{~kb}$ downstream of hairy. (B) The close up of the hairy downstream enhancer region shows FOXO, HIF-1 and HSF binding sites as well as coincidence with a ncRNA that is also up-regulated under hypoxia. 


\section{Table $\mathbf{1}$ (on next page)}

\section{Properties of Discovered Hypoxic Enhancers}

The 31 hypoxic enhancers identified by our genome-wide screen are shown in order of statistical significance. Column one is the genomic location of the enhancer ( $\mathrm{dm} 3)$. Column two is the p-value between hypoxic and normoxic counts as calculated by the negative binomial test with column three showing the Benjamini-Hochberg adjusted p-value. Column four is the fold change of transcriptional activity due to the enhancer in hypoxic versus normoxic conditions. Column five shows endogenous genes within $20 \mathrm{~kb}$ that were significantly up-regulated under hypoxia in the same RNA extracts used to calculate enhancer activity. The rank of the gene's hypoxic induction is shown in parentheses and genes marked with an asterisk were observed to be up-regulated under hypoxia in Drosophila by Li et al. ${ }^{27}$. Column six indicates the relative position of the enhancer to the proximal hypoxic up-regulated gene. Column seven shows binding sites for stress-related transcription factors found in the enhancer. 


\begin{tabular}{|c|c|c|c|c|c|c|}
\hline Enhancer Locus & P-value & $\begin{array}{l}\text { Adjusted } \\
\text { P-value }\end{array}$ & $\begin{array}{l}\text { Fold } \\
\text { Change }\end{array}$ & $\begin{array}{l}\text { Hypoxic Gene(s) } \\
\text { Within 20Kb }\end{array}$ & $\begin{array}{l}\text { Relative Position to } \\
\text { Hypoxic Gene(s) }\end{array}$ & $\begin{array}{l}\text { Stress TF } \\
\text { Binding Sites }\end{array}$ \\
\hline 3R:8303000..8303500 & $7.79 \mathrm{e}-22$ & $4.63 \mathrm{e}-16$ & 5.08 & Hsp70B genes (1-4) & Intergenic & Hsf, Hif-1, Foxo \\
\hline 3L:6256700..6257200 & $1.83 \mathrm{e}-16$ & $2.72 \mathrm{e}-11$ & 5.95 & impl3 (9) & Upstream & NF-kB \\
\hline 3R:8331100..8331800 & $1.59 \mathrm{e}-16$ & $2.72 \mathrm{e}-11$ & 4.49 & Hsp70Bb (2) & Promoter Proximal & Hsf, Hif-1, Foxo \\
\hline 3R:8293200..8293900 & $2.96 \mathrm{e}-16$ & $3.51 \mathrm{e}-11$ & 3.83 & Hsp70Ba (4) & Promoter Proximal & Hsf, Hif-1, Foxo \\
\hline 3R:8334400..8335000 & $1.18 \mathrm{e}-15$ & $1.01 \mathrm{e}-10$ & 4.45 & Hsp70Bc (1) & Promoter Proximal & Hsf, Hif-1, Foxo \\
\hline 2L:8001300..8001800 & $2.64 \mathrm{e}-15$ & $1.74 \mathrm{e}-10$ & 6.44 & Wwox (15) & Intronic & Hif-1 \\
\hline $3 \mathrm{R}: 8327800 . .8328500$ & 8.89 e- 13 & $2.40 \mathrm{e}-08$ & 3.70 & Hsp70Bbb (3) & Promoter Proximal & Hsf, Hif-1, Foxo \\
\hline 2L:20082900..20083500 & $1.08 \mathrm{e}-12$ & $2.79 \mathrm{e}-08$ & 6.35 & Fok (11) & Intronic & Foxo, Hif-1 \\
\hline 3L:8685300..8685800 & $1.07 \mathrm{e}-10$ & $2.18 \mathrm{e}-06$ & 3.79 & Hairy (45) & Downstream & Hsf, Hif-1, Foxo \\
\hline 3L:7797800..7798600 & $1.77 \mathrm{e}-10$ & $3.38 \mathrm{e}-06$ & 3.07 & CG32369 (23) & Intronic & Hif-1 \\
\hline 3L:9385200..9385800 & $2.14 \mathrm{e}-09$ & $3.62 \mathrm{e}-05$ & 3.71 & $\begin{array}{l}\text { Hsp22,23,26,27 } \\
(7,8,10,14)\end{array}$ & Neighboring Intron & Not Detected \\
\hline $\mathrm{X}: 17071000 . .17071300$ & $8.77 \mathrm{e}-09$ & $1.24 \mathrm{e}-04$ & 4.99 & Not Detected & Not Detected & Not Detected \\
\hline X:9767000..9767500 & $1.27 \mathrm{e}-08$ & $1.76 \mathrm{e}-04$ & 3.65 & CG32695* & ORF & Not Detected \\
\hline 2L:2887100..2887600 & $1.32 \mathrm{e}-08$ & $1.79 \mathrm{e}-04$ & 5.82 & Not Detected & Not Detected & Hif-1 \\
\hline 3L:11234100..11234900 & $6.03 \mathrm{e}-07$ & $6.63 \mathrm{e}-03$ & 2.68 & Scylla (19) & Upstream & Foxo \\
\hline 3L:3892900..3893100 & $1.55 \mathrm{e}-06$ & $1.59 \mathrm{e}-02$ & 2.75 & Not Detected & Not Detected & Hif-1, NF-kB \\
\hline 2L:5986900..5987500 & $1.82 \mathrm{e}-06$ & $1.81 \mathrm{e}-02$ & 2.16 & ifc* & Intronic & Foxo \\
\hline 3L:9448800..9448900 & $2.09 \mathrm{e}-06$ & $2.03 \mathrm{e}-02$ & 5.39 & MTF-1* & Neighboring Intron & NF-kB, Hif-1 \\
\hline 3R:6800900..6801600 & $2.22 \mathrm{e}-06$ & $2.09 \mathrm{e}-02$ & 13.82 & Not Detected & Not Detected & Hif-1 \\
\hline 3L:11522800..11523300 & $2.66 \mathrm{e}-06$ & $2.35 \mathrm{e}-02$ & 3.04 & Not Detected & Not Detected & NF-kB \\
\hline 3R:4181100..4181600 & $2.66 \mathrm{e}-06$ & $2.35 \mathrm{e}-02$ & 3.87 & $\operatorname{Atg} 13(51)$ & Downstream & Foxo, Hif-1 \\
\hline 3R:7781900..7782700 & $2.69 \mathrm{e}-06$ & $2.35 \mathrm{e}-02$ & 4.96 & Hsp70Aa (6) & Promoter Proximal & Hsf \\
\hline 3R:7783900..7784500 & $2.75 \mathrm{e}-06$ & $2.37 \mathrm{e}-02$ & 4.18 & Hsp70Ab (5) & Promoter Proximal & Hsf \\
\hline 3R:21433600..21434000 & $3.30 \mathrm{e}-06$ & $2.72 \mathrm{e}-02$ & 9.03 & Not Detected & Not Detected. & Not Detected \\
\hline $\mathrm{X}: 16559200 . .16559700$ & $4.13 \mathrm{e}-06$ & $3.23 \mathrm{e}-02$ & 6.56 & Not Detected & Not Detected & Foxo \\
\hline $3 R: 2902300 . .2902600$ & $6.21 \mathrm{e}-06$ & $4.63 \mathrm{e}-02$ & 2.95 & Not Detected & Not Detected & Not Detected \\
\hline 2R:12896000..12896500 & $6.88 \mathrm{e}-06$ & $5.05 \mathrm{e}-02$ & 3.02 & Not Detected & Not Detected & Foxo \\
\hline $\mathrm{X}: 17388000 . .17388500$ & $8.24 \mathrm{e}-06$ & $5.75 \mathrm{e}-02$ & 6.80 & Not Detected & Not Detected. & Hif-1 \\
\hline 3R:14892300..14892800 & $9.76 \mathrm{e}-06$ & $6.44 \mathrm{e}-02$ & 18.01 & Not Detected & Not Detected & Hif-1 \\
\hline 3R:27050000..27050500 & $1.52 \mathrm{e}-05$ & $9.40 \mathrm{e}-02$ & 2.78 & CG12054* & Intronic & Hif-1 \\
\hline 3R:25921500..25922100 & $1.54 \mathrm{e}-05$ & $9.44 \mathrm{e}-02$ & 2.46 & Hif-1 (71) & Intronic & NF-kB, Hif-1 \\
\hline
\end{tabular}




\section{Table 2 (on next page)}

ncRNAs Proximal to Hypoxic Enhancers

Three of the five enhancers not contained within protein coding transcripts coincide with ncRNAs. Each of these ncRNAs is also up-regulated under hypoxia. 
1

\begin{tabular}{|l|l|l|l|l|}
\hline Enhancer Locus & ncRNA & $\begin{array}{l}\text { Position of ncRNA } \\
\text { relative to enhancer }\end{array}$ & $\begin{array}{l}\text { Hypoxic read } \\
\text { counts }\end{array}$ & $\begin{array}{l}\text { Normoxic } \\
\text { read counts }\end{array}$ \\
\hline 3R:8303000..8303500 & CR32865 & overlapping & 66 & 13 \\
\hline 3L:8685300..8685800 & CR44526 & 3 bp upstream & 31 & 14 \\
\hline 3L:6256700..6257200 & CR44522 & 201 bp upstream & 6 & 1 \\
\hline
\end{tabular}

2 


\section{Table 3 (on next page)}

P-value of Stress Transcription Factor Binding Site Enrichment in Discovered Enhancer Sequences 
1

\begin{tabular}{|l|l|}
\hline $\begin{array}{l}\text { Transcription } \\
\text { Factor }\end{array}$ & $\begin{array}{l}\text { P-value of } \\
\text { Enrichment }\end{array}$ \\
\hline HSF & 6.22 e-12 \\
\hline Hif-1 & 6.49 e-06 \\
\hline Foxo & 1.01 e-04 \\
\hline NF-kB & 6.67 e-04 \\
\hline \multicolumn{2}{|l}{2}
\end{tabular}

